\title{
Partial reproductive isolation between European subspecies of honey bees
}

\author{
Andrzej Oleksa ${ }^{1}$, Jerzy WiLde ${ }^{2}$, Adam Tofilski ${ }^{3}$, Igor J. ChYBicki ${ }^{1}$ \\ ${ }^{1}$ Department of Genetics, Kazimierz Wielki University, Chodkiewicza 30, 85-064 Bydgoszcz, Poland \\ ${ }^{2}$ Apiculture Division, Warmia and Mazury University, Słoneczna 48, 10-710 Olsztyn, Poland \\ ${ }^{3}$ Department of Pomology and Apiculture, Agricultural University, 29 Listopada 54, 31-425 Kraków, Poland
}

Received 23 November 2012 - Revised 4 April 2013 - Accepted 17 April 2013

\begin{abstract}
Northern Poland is inhabited by native Apis mellifera mellifera (AMM) and the non-native A. $m$. carnica (AMC) which was introduced by beekeepers. However, hybrids between the two subspecies of honey bee are relatively rare. The lower than expected proportion of hybrids is hypothesised to be related to reproductive isolation between AMM and AMC. To verify this hypothesis, we allowed the AMM and AMC queens to be naturally inseminated in an area inhabited by both AMM and AMC drones. Genotype of the queens and their sexual partners were derived based on random samples of their worker offspring. Assignment of parental genotypes to the two subspecies was performed with a Bayesian clustering method. In colonies headed by AMM queens, workers were fathered mainly by AMM drones. On the other hand, in colonies headed by AMC queens workers were fathered by drones of both subspecies. The partial reproductive isolation reported here between AMM and AMC may facilitate conservation of the declining population of AMM.
\end{abstract}

Apis mellifera mellifera / Apis mellifera carnica / reproductive isolation / assortative mating / parentage analysis

\section{INTRODUCTION}

The honey bee (Apis mellifera) is a species with a wide geographical range and considerable variation. Based on morphology and molecular data, up to 30 subspecies of honey bees are distinguished (Ruttner 1988; De la Rúa et al. 2009). Probably, all the subspecies are capable of interbreeding and producing fertile offspring, at least under instrumental insemination (Koeniger and Koeniger 2000). It is not clear how various subspecies maintain their distinctiveness in hybrid zones, where ranges of two subspecies come in contact without geographical barriers (Badino et al.

Corresponding author: A. Oleksa, olek@ukw.edu.pl Manuscript Editor: Marina Meixner
1982; Nazzi 1992). This may be related to partial reproductive isolation between the subspecies (Koeniger et al. 1989) or low fitness of hybrids (Beekman et al. 2007, 2012).

In Europe, there are two distinct evolutionary lineages of honey bees: M and C (Ruttner 1988; Meixner et al. 2007). The existence of these two divergent evolutionary units results from two independent colonisation events (Garnery et al. 1992; Han et al. 2012). The contact between both lineages was established along the mountain ranges of central Europe, i.e. the Alps and Carpathians (Ruttner 1988). The presence of the hybrid zone between $\mathrm{M}$ and $\mathrm{C}$ lineages in this area was confirmed by Meixner et al. (2007). If such a contact zone is to be maintained, restrictions to free gene flow between both populations would be necessary. 
Northern Poland was originally inhabited by A. m. mellifera (hereafter AMM) representing the $\mathrm{M}$ lineage. However, most beekeepers in this area prefer non-native $A$. $m$. carnica (hereafter AMC), representing the $\mathrm{C}$ lineage, which led to a massive import of this subspecies (Meixner et al. 2010, Semkiw and Skubida 2010). Given high dispersal rates (Schmidt 1995, Mistro et al. 2005) and short generation times of honey bees, several decades of active propagation of $\mathrm{AMC}$ in the natural range of AMM would seem enough time for complete hybridisation of the two subspecies. Nonetheless, in some parts of Poland, relatively little admixture of AMM populations has been reported (Meixner et al. 2007; Oleksa et al. 2011). Earlier studies (Soland-Reckeweg et al. 2009) and our preliminary observations (unpublished data) showed that hybrids of AMM and $\mathrm{AMC}$ are relatively rare even if the two subspecies are kept in sympatry by beekeepers. The relatively low frequency of hybrids indicates limited gene flow between the two subspecies. This may result from partial reproductive isolation between the subspecies or low fitness of hybrids. The reproductive isolation can be related to preferential mating between queens and drones of the same subspecies. Partial reproductive isolation has been already observed in some honey bee subspecies (Kerr and Bueno 1970; Koeniger et al. 1989; Holmes et al. 2010).

In this study, we demonstrate evidence for reproductive isolation between AMM and AMC. In an area inhabited by both AMM and $\mathrm{AMC}$, we introduced unmated queens of the two subspecies and determined the presence of hybrids in their offspring. We expected workers to be fathered more often by drones of the same subspecies as the mother queen.

\section{MATERIALS AND METHODS}

\subsection{Design of experiment}

The experimental site was situated in the vicinity of Rychliki in northern Poland, $53^{\circ} 58^{\prime} 23^{\prime \prime} \mathrm{N}$, $19^{\circ} 35^{\prime} 44^{\prime \prime} \mathrm{E}$. This area is a typical postglacial land- scape with relatively fertile soils developed on moraines. Agriculture is the predominat form of land use (74\% of the municipality of Rychliki), while highly fragmented forests cover $17 \%$. Beekeeping is well established in this area, and the density of managed bee populations is estimated to be 4.4 colonies $/ \mathrm{km}^{2}$ (Semkiw and Skubida 2010). There is also a viable feral population of honey bees inhabiting hollow trees (Oleksa et al. 2013). The density of the feral population was estimated as 0.1 colonies/ $\mathrm{km}^{2}$. Most beekeepers declare that they keep AMC, while our preliminary studies show that most colonies belong to the native AMM (unpublished data).

The experiment was carried out between 25 June and 11 August 2011. We used 48 virgin queens (24 $\mathrm{AMM}$ and $24 \mathrm{AMC}$ ) which were reared from progeny of 6 mother queens ( $3 \mathrm{AMM}$ and $3 \mathrm{AMC}$ ). From each mother queen, 8 virgin queens were obtained. The AMM queens were from the Augustowska breeding line preserved in a closed area for breeding (described by Oleksa et al. 2011), while the AMC queens were from the commercially available breeding line Kortówka (Costa et al. 2012). The virgin queens were introduced into mini-plus nuclei with six combs and about 2,500 AMC workers (Siuda et al. 2011). Soon after the queens started egg laying, their sealed brood was moved into an incubator $\left(34.5{ }^{\circ} \mathrm{C}\right)$. The emerged workers were preserved in absolute ethanol. For the analysis, 8 workers per colony were used. Until extraction, samples were stored in $-20{ }^{\circ} \mathrm{C}$.

Additionally, we examined the gene pool of the local honey bee population, which was the putative source of drones inseminating the queens. For this purpose, we sampled 100 colonies from three neighbouring apiaries $(38,32$ and 30 colonies from each apiary, respectively; each colony represented by one worker).

\subsection{Molecular analyses}

Total genomic DNA was extracted from insect thoraxes with EZNA Insect Easy DNA Kit (Omega Bio-Tek, Norcross, GA, USA) according to the standard protocol of the manufacturer, and then subjected to PCR (polymerase chain reaction) treatment. In the study, we used 17 nuclear microsatellite loci (Solignac et al. 2003), amplified in two 
multiplex reactions: multiplex 1: A113, A24, A7, A88, Ap28, Ap43, Ap55 and Ap66; multiplex 2: A025, Ac011, Ap090, Ap103, Ap226, Ap238, Ap243, Ap249 and Ap256. Forward primers for these loci were 5'-labelled with fluorescent dyes to enable their simultaneous detection during capillary electrophoresis. Multiplex PCR was performed using the Multiplex PCR Kit (QIAGEN, Hilden, Germany) following the recommended protocol in a final reaction volume of $10 \mu \mathrm{L}(5 \mu \mathrm{L}$ of $2 \times$ QIAGEN Multiplex Master Mix, $4 \mu \mathrm{L}$ of primer mix and $1 \mu \mathrm{L}$ of template DNA). The PCR cycling started with an initial incubation at $95{ }^{\circ} \mathrm{C}$ for $15 \mathrm{~min}$. It was followed by nine touchdown cycles of $94{ }^{\circ} \mathrm{C}$ for $30 \mathrm{~s}, 60^{\circ} \mathrm{C}$ $\left(-0.5{ }^{\circ} \mathrm{C}\right.$ per cycle) for $1 \mathrm{~min} 30 \mathrm{~s}$ and $72{ }^{\circ} \mathrm{C}$ for $1 \mathrm{~min}$, and 24 cycles of $94{ }^{\circ} \mathrm{C}$ for $30 \mathrm{~s}, 55^{\circ} \mathrm{C}$ for $1 \mathrm{~min} 30 \mathrm{~s}$ and $72{ }^{\circ} \mathrm{C}$ for $1 \mathrm{~min}$. Finally, tubes were incubated at $72{ }^{\circ} \mathrm{C}$ for $10 \mathrm{~min}$. Amplifications were carried out using PTC200 thermocycler (BioRad, Hercules, CA, USA).

The separation of fragments was carried out on automated sequencer ABI PRISM 3130xl (Applied Biosystems, Foster City, CA, USA) using the internal size standard (LIZ 600; Applied Biosystems). Resulting electropherograms were scored using GeneScan v.3.7 and Genotyper v.3.7 software (Applied Biosystems).

\subsection{Statistical procedures}

Maternal (diploid) and paternal (haploid) genotypes were inferred for each colony based on random samples of 8 worker genotypes. For this purpose, we used a Bayesian approach elaborated originally to estimate heterogeneity among pools of parental gametes (Chybicki 2013). The method assumes data consisting of progeny groups, each originated from mating between a single mother and a number of fathers. Paternal populations might differ among families, so that each family can reveal a degree of (unknown and estimable) divergence from a base population of fathers. To account for divergence, the $F$ model (reviewed in Gaggiotti and Foll 2010) is assumed as a prior distribution for paternal allele frequencies. For the estimation, we used the Gibbs sampler (Hoff 2009). Because the originally developed method assumes maternal genotypes to be known, here we used the refined algorithm which allows maternal genotypes to be unknown and estimable quantities. For this purpose, we developed the specific sampling scheme, described in detail in the Appendix. Genotypes were inferred using a Pascal program (MSF; available from IJC upon request), based on 50,000 iterations, rejecting the first 10,000 for burn-in. Because queen genotypes were identified based on relatively small samples of offspring, there is a probability of not detecting one of two queen alleles (when a queen is heterozygous), equal to $\varepsilon=0.5^{\wedge}(n-1)$ [ $n$ (number of offspring genotypes) $=8$ ]. In this study, the average $\varepsilon$ equalled to 0.00781 , allowing us to conclude that sampling of a limited number of workers had a negligible effect on the observed heterozygosities.

General characteristics of genetic diversity (allele frequencies, observed and expected heterozygosity, and polymorphism information content) for the parental population were computed using GenAlEx (Peakall and Smouse 2006).

Assignment of parental genotypes to the two subspecies (AMM and AMC) was performed with Bayesian clustering implemented in the InStruct software (Gao et al. 2007). The method assumes that there are $K$ populations, each of which is characterised by a set of allele frequencies at each locus. Individuals in the sample are probabilistically assigned to one of the populations, or jointly to two or more populations, if their genotypes indicate they are admixed. The Markov Chain Monte Carlo method allows the posterior probability distribution to be computed for estimated parameters. This method is similar to the widely used STRUCTURE algorithm (originally described in Pritchard et al. 2000); however, it does not account for the presence of Hardy-Weinberg and linkage disequilibrium. We preferred InStruct to STRUCTURE because our data were partially composed of haploid drones, which were treated as homozygotes carrying two copies of each allele at each locus. This resulted in an excess of homozygous genotypes and the violation of the assumptions of Hardy-Weinberg model. It should be noted, however, that analysis with STRUCTURE resulted in almost identical results (not presented or discussed here to limit the length of this paper). InStruct allows for different assumptions on the ancestry of the population (i.e. ancestral populations could be admixed or not admixed). We used the 
admixture model, which assumes that each individual (i) has inherited some fraction of its genome from ancestors in all $K$ populations, and the correlated allele frequency model. A burn-in of 100,000 iterations, followed by $1,000,000$ iterations was applied. In the analysis, queen and drone genotypes, inferred from workers' genotypes (see above), were included. Haploid males were treated as homozygotes carrying two copies of each allele at each locus. Individual assignment probabilities to AMM, $q_{A M M}$, together with their variances, were estimated for each genotype. Studied bees were expected to belong to two distinct groups (the two subspecies); however, to verify this assumptions, the ad hoc statistic of $\Delta K$ (Evanno et al. 2005) was used to find the most likely uppermost level of structure. Five runs for each $K$ value, ranging from 1 to 5 , were used. This analysis confirmed $K=2$ as the most likely number of populations that has contributed to the gene pool of the studied bees. We thus report results only for $K=2$.

The relationship between the subspecific assignment of the queens and assignment of their sexual partners (median computed over the groups of drones) was assessed by Spearman's rank correlation and regression analysis. We preferred reduced major axis to ordinary least square regression because both variables are measured with error and distribution of errors is symmetrical (Smith 2009), as is the case of assignment probabilities based on genotype clustering. In order to assess confidence intervals for a slope and intercept coefficient, we performed bootstrap analyses based on assignment probabilities of drones within families taken as independent replicates. The procedure relied on drawing (with replacement) a random sample of drones from a given family, for which median value of assignment probability was estimated. Then, bootstrap medians were used along with (fixed) assignment probabilities for the queens to compute bootstrap slope and intercept. $95 \%$ confidence intervals were obtained based on 10,000 bootstrap replicates. Comparison of assignment probabilities between groups of bees included in the experiment were performed with Mann-Whitney $U$ tests with Bonferroni correction for multiple comparisons. Calculations (except for bootstrap) were made with PAST data analysis package (Hammer et al. 2012). Bootstrap procedure was implemented in ad hoc Pascal computer program.

\section{RESULTS}

The queens returned from the nuptial flights and started to lay eggs in all but three cases (all in AMM). Therefore, the analyses were based on $24 \mathrm{AMC}$ and $21 \mathrm{AMM}$ colonies.

All 17 microsatellite loci were moderately to highly polymorphic in the worker progeny, providing useful information for inferring parental genotypes (for detailed information on characteristics of genetic diversity, see supplementary online resources)

In the total sample of inferred queen genotypes, there were 115 alleles at 17 loci, where 49 alleles could be described as rare, with a frequency of $5 \%$ or less $\left(p_{i} \leq 0.05\right)$. The number of alleles was higher in AMM queens (98 alleles, including 54 rare alleles) than in $\mathrm{AMC}$ queens (91 alleles, 62 rare alleles). In the total paternal population (drones that mated with AMM and AMC), there were 144 alleles (86 rare alleles). Sexual partners of AMC were more diverse in terms of allelic richness than those of AMM (133 and 113 alleles, respectively, with 82 and 86 rare alleles).

Queens of both subspecies showed similar levels of observed heterozygosity $(0.59 \pm 0.05$ in AMC vs. $0.61 \pm 0.03$ in AMM). Comparison of observed and estimated heterozygosities (values of $H_{e} \quad 0.54 \pm 0.05$ and $0.56 \pm 0.03$, respectively) showed that there was significant heterozygote excess in both subspecies (values of $F$ were $-0.09 \pm$ 0.03 and $-0.08 \pm 0.03$, respectively). No departures from Hardy-Weinberg equilibrium were detected, except for two loci in AMM (Ap243 and Ap256; Table I).

Bees from nearby apiaries were not a homogeneous group, as indicated by the signs of Wahlund effect, i.e. reduction of heterozygosity $(F=0.16 \pm 0.03)$ and deviations from Hardy-Weinberg equilibrium (in 7 of 17 loci) caused by the presence of subpopulation structure (Online Resource 1). Although most of these bees were unambiguously classified as AMM by InStruct analysis, there were also AMCs, probably as a result of recent importation of queens (as claimed by beekeepers). 
Table I. Proportion of genes of Apis mellifera mellifera $\left(q_{A M M}\right)$ estimated by InStruct clustering (medians with 25-75\% percentiles) and pairwise differences between studied groups of bees (significance of Mann-Whitney $U$ test for differences between pairs of groups, Bonferroni corrected).

\begin{tabular}{|c|c|c|c|c|c|c|c|}
\hline \multirow[t]{2}{*}{ Group } & \multirow[t]{2}{*}{$n$} & \multirow[t]{2}{*}{$q_{\mathrm{AMM}}$} & \multicolumn{5}{|c|}{ Mann-Whitney pairwise comparisions } \\
\hline & & & 1 & 2 & 3 & 4 & 5 \\
\hline 1. AMC queens & 24 & $0.015(0.003,0.080)$ & & & & & \\
\hline 2. Drones inseminating $\mathrm{AMC}$ queens & 184 & $0.811(0.019,0.991)$ & $* * *$ & & & & \\
\hline 3. AMM queens & 21 & $0.882(0.661,0.994)$ & $* * *$ & ns & & & \\
\hline 4. Drones inseminating AMM queens & 156 & $0.991(0.842,0.996)$ & $* * *$ & $* * *$ & ns & & \\
\hline 5. Workers from nearby apiaries & 100 & $0.865(0.395,0.989)$ & $* * *$ & ns & ns & *** & \\
\hline
\end{tabular}

ns no significant difference, AMC A. m. carnica, AMM A. m. mellifera

*** significant at $p<0.001$

Among 100 examined colonies from local apiaries, 19 did not differ significantly from $\mathrm{AMC}, 58$ did not differ from AMM and the rest (23 colonies) represented hybrids. The median proportion of AMM genes in the genetic pool of local bees amounted to

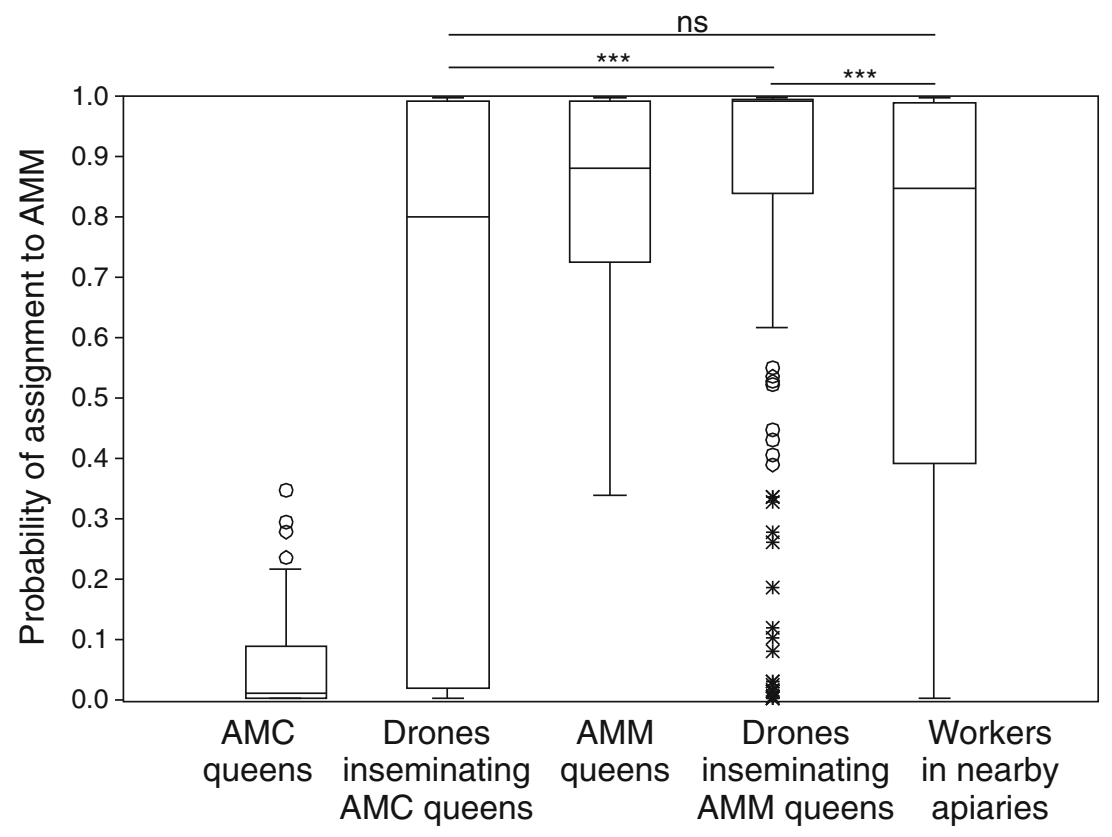

Figure 1. Comparison of assignment probabilities of queens (AMC A. m. carnica, AMM A. m. mellifera), their sexual partners and bees from nearby apiaries. For each sample, the $25-75 \%$ quartiles are drawn using a box. The median is shown with a horizontal line inside the box. The whiskers are drawn from the top of the box to the largest data point less than 1.5 times the box height above the box (the "upper inner fence"), and similarly below the box. Values outside the inner fences are shown as circles, values further than 3 times the box height from the box (the "outer fences") are shown as stars. Horizontal lines indicate pairwise differences between studied groups of bees (significance of Mann-Whitney $U$ test for differences between pairs of groups, Bonferroni corrected, $n s$ not significant, ${ }^{* * *} p<0.001$ ). 
$86.5 \%$ (Online Resource 2). If there is random mating, it could be expected that drones inseminating AMC and AMM queens should exhibit similar assignment coefficient as these local colonies (Fig. 1). The two groups of drones showed, however, directional deviations towards proportions detected in AMC and AMM queens. Especially, drones inseminating AMM had a higher proportion of AMM genes than bees from local apiaries $(P<0.0001$, Mann-Whitney $U$ test; Table I), while the difference between drones inseminating AMC queens and bees from local apiaries was insignificant.

Assignment probabilities of maternal genotypes positively correlated with the median assignment of their sexual partners (Spearman's rank correlation, $r_{s}=0.589, n=45, P=0.0004$; Fig. 2). Progeny of AMM queens was fathered almost exclusively by drones from their own subspecies. However, progeny of AMC queens was fathered by drones from both subspecies. In the case of AMM, $95 \%$ bootstrap confidence intervals around slope coefficient did not include zero $(0.422,1.071)$, revealing a positive association between assignment probabilities of queens and drones. In contrast, in AMC, the slope coefficient was not significantly different from zero (95 \% CI: $-3.056,3.901)$.

\section{DISCUSSION}

The results presented here suggest partial reproductive isolation between AMM and AMC. The isolation is asymmetrical. The progeny of AMM queens was fathered almost exclusively by AMM drones. On the other hand, progeny of AMC queens was fathered by drones of both subspecies. The drones which fathered AMC progeny tended to be more similar to AMC than drones in nearby apiaries, though the difference was not significant. The results could be affected to some degree by lower availability of AMC drones in the study area, It is possible that in presence of equal proportion of drones of both subspecies, AMC queens would show higher preference to drones of the same subspecies. In order to solve this problem, an experiment should be conducted in an isolated mating site where the presence of drones can be controlled. However, the large difference between subspecific assignment of drones which fathered progeny of AMM and AMC queens provides evidence for partial reproductive isolation between the two subspecies.

The asymmetry of reproductive isolation between AMM and AMC agrees with the reinforcement hypothesis suggesting that reproductive isolation would evolve in zones of overlap between subspecies (Howard 1993). Such reinforcement is more likely to evolve in the Augustowska breeding line of AMM. This breeding line is largely under natural selection and in constant risk of hybridisation with AMC. On the other hand, the commercial breeding lines of AMC are under artificial selection and there is no risk of hybridisation with AMM because they are maintained by instrumental insemination.

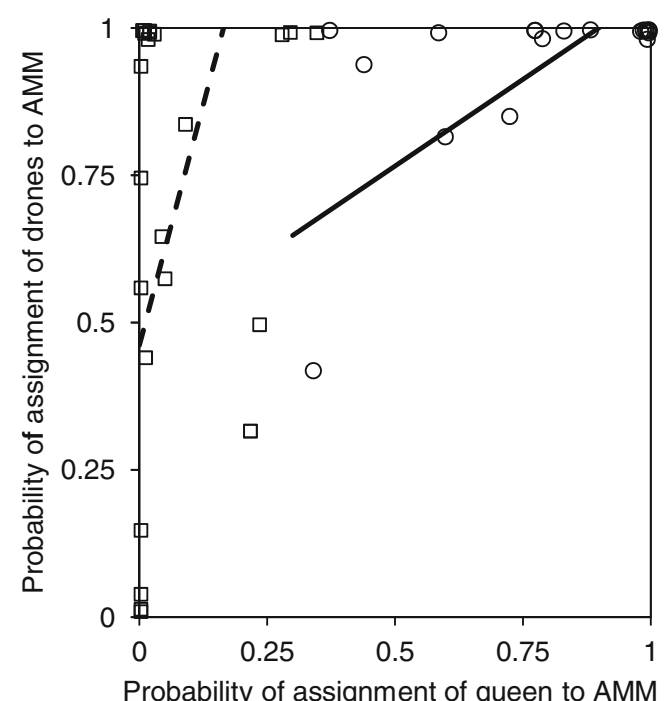

Figure 2. Relationship between assignment probabilities of queens and median assignment of their sexual partners (values represent probability of being $A . m$. mellifera). Circles indicate A. m. mellifera (AMM) and squares A. m. carnica (AMC). For both groups, regression lines were fitted with reduced major axis method: AMC dotted line, $y=3.26 x+0.46, R^{2}=0.04$, $P=0.37$; AMM solid line, $y=0.58 x+0.47, R^{2}=0.34$, $P=0.005$. 
The mechanisms of reproductive isolation can be related to both prezygotic and postzygotic barriers (reviewed by Koeniger and Koeniger 2000). The prezygotic barriers are differences between subspecies in: location of drone congregation areas, time of mating flights, shape and size of copulatory organs or survival of spermatozoa in spermatheca (Phiancharoen et al. 2004). There is some evidence concerning the prezygotic barriers between honey bee subspecies. AMC drones search for queens at a higher altitude than A. m. ligustica drones (Koeniger et al. 1989). On the other hand, the differences in time of day between honey bee subspecies when drones perform mating flights are small (Drescher 1969; Tuchashvili 1969; Rowell et al. 1986) and probably not important for the reproductive isolation. There are, however, differences in this respect between two colour morphs of $A$. cerana (Oldroyd et al. 2006). In contrast to the prezygotic barriers mentioned above, there is no information about postzygotic barriers between honey bee subspecies - the hybrids, for example the Buckfast breading line, are both viable and fertile.

A lower than expected proportion of hybrids in places where AMM and AMC are kept in sympatry (Soland-Reckeweg et al. 2009; own observations) can be related not only to the reproductive isolation but also to the lower fitness of the hybrids. In the warmer coastal regions of Tasmania, AMM readily hybridised with $A . m$. ligustica, but in cooler mountain regions there was much less hybridisation and AMM was more abundant (Oldroyd et al. 1995). Selective survival of subspecies in various environments was also observed in South America where African and European honey bee subspecies have been introduced (Sheppard et al. 1991).

The partial reproductive isolation reported here between AMM and AMC may facilitate the protection of AMM. Even in the absence of spatial isolation, a relatively large proportion of colonies can be maintained pure. Moreover, hybrids and non-native bees can be further eliminated by natural selection, as pure AMM may be better adapted to the environment within its native range.

\section{ACKNOWLEDGEMENTS}

We thank Henryk Kiejdo for his help with performing the field experiment and Katarzyna Meyza and Ewa Sztupecka for their laboratory work. The study was partially supported by grants from Polish Ministry of Science and Higher Education (grant no. N N311 509639) to A.O. and by the Research Project of UWM in Olsztyn, Poland No 528.0113.0811.

Isolation reproductive partielle entre les sousespèces d'abeilles européennes

Apis mellifera mellifera / Apis mellifera carnica / isolation reproductive / homogamie/analyse de parenté

\section{Partielle reproduktive Isolation zwischen europäischen Unterarten der Honigbiene}

Apis mellifera mellifera / Apis mellifera carnica / assortative Paarung / reproduktive Isolation / Vaterschaftsanalyse

Open Access This article is distributed under the terms of the Creative Commons Attribution License which permits any use, distribution, and reproduction in any medium, provided the original author(s) and the source are credited.

\section{Appendix}

Here, we explain details of the sampling scheme used for the inference of maternal genotypes, given half-sib progeny samples. As in Chybicki (2013), we denote the conditional probability for the $j$-th offspring's genotype present in the $i$-th family as $\operatorname{Pr}\left(O_{i j} \mid M_{i},\left\{p_{i l m}\right\}\right)$, where $M_{i}$ is a genotype of the mother common to all offspring in the $i$-th family and $\left\{p_{i l m}\right\}$ is a set of allele frequencies in a subpopulation of fathers $\left(l=1 \ldots L, m=1 \ldots M_{l}\right.$, where $L-$ a number of loci, $M_{l}-$ a number of alleles at the $l$-th locus). The likelihood for the $i$-th family is then

$L\left(\left\{O_{i j}\right\} ; M_{i},\left\{p_{i l m}\right\}\right)=\Pi_{j} \operatorname{Pr}\left(O_{i j} \mid M_{i},\left\{p_{i l m}\right\}\right)$.

In order to sample $M_{i}$, we used the Metropolis algorithm, taking a categorical uniform distribution as a prior for $M_{i}$. Given the set of 
parameter values at the $s$-th iteration $\left(M_{i}^{(s)}\right.$, $\left.\left\{p_{i l m}\right\}^{(s)}\right)$, new maternal genotype for the $i$-th family was obtained as follows:

1. Propose new maternal genotype $\left(M_{i}^{*}\right)$ : At the $l$-th randomly chosen locus, one randomly chosen allele in maternal genotype was replaced with another allelic state randomly selected (with replacement) out of $M_{l}$ allelic states available at that locus.

2. Compute the acceptance ratio. Given the uniform prior and the symmetric proposal distribution, the acceptance ratio equals: $R=\frac{L\left(\left\{O_{i j}\right\} ; M_{i}^{*},\left\{p_{i l m}\right\}^{(s)}\right)}{L\left(\left\{O_{i j}\right\} ; M_{i}^{(s)},\left\{p_{i l m}\right\}^{(s)}\right)}$

3. Update maternal genotype: $M_{i}^{(s+1)}=M_{i}^{*}$ with the probability $\min (R, 1)$ and $M_{i}^{(s+1)}=M_{i}^{(s)}$ otherwise.

Maternal genotypes were updated cyclically, together with the remaining parameters of the model (see Chybicki 2013).

\section{REFERENCES}

Badino, G., Celebrano, G., Manino, A. (1982) Genetic variability of Apis mellifera ligustica Spin. in a marginal area of its geographical distribution. Experientia 38, 540-541

Beekman, M., Allsopp, M.H., Holmes, M.J., Lim, J., Noach-Pienaar, L.A., Wossler, T.C., Oldroyd, B.P. (2012) Racial mixing in South African honeybees: the effects of genotype mixing on reproductive traits of workers. Behav. Ecol. Sociobiol. 66, 897-904

Beekman, M., Allsopp, M.H., Wossler, T.C., Oldroyd, B.P. (2007) Factors affecting the dynamics of the honeybee (Apis mellifera) hybrid zone of South Africa. Heredity 100, 13-18

Chybicki, IJ (2013) Note on the applicability of the Fmodel in analysis of pollen pool heterogeneity. J. Heredity. doi:10.1093/jhered/est029

Costa, C., Büchler, R., Berg, S., Bienkowska, M., Bouga, M., et al. (2012) A Europe-wide experiment for assessing the impact of genotype-environment interactions on the vitality of honey bee colonies: Experimental design and trait evaluation. J. Apic. Sci. 56, 147-158

De la Rúa, P., Jaffé, R., Dall'Olio, R., Muñoz, I., Serrano, J. (2009) Biodiversity, conservation and current threats to European honeybees. Apidologie 40, 263-284
Drescher, W. (1969) Die Flugaktivität von Drohnen der Rasse Apis mellifica carnica L und Apis mellifera ligustica L. in Abhängigkeit von Lebensalter und Witterung. Z. Bienenforsch. 9, 390-409

Evanno, G., Regnaut, S., Goudet, J. (2005) Detecting the number of clusters of individuals using the software STRUCTURE: a simulation study. Mol. Ecol. 14, 2611-2620

Gaggiotti, O., Foll, M. (2010) Quantifying population structure using the F-model. Mol. Ecol. Res. 10, 821-830

Gao, H., Williamson, S., Bustamante, C.D. (2007) A Markov chain Monte Carlo approach for joint inference of population structure and inbreeding rates from multilocus genotype data. Genetics 176, 1635-1651

Garnery, L., Cornuet, J.M., Solignac, M. (1992) Evolutionary history of the honey bee Apis mellifera inferred from mitochondrial DNA analysis. Mol. Ecol. 1, 145-154

Hammer, Ø., Harper, D.A.T., Ryan, P.D. (2012) PAST: Paleontological Statistics Software Package for Education and Data Analysis. Palaeontol. Electron. 4, 1-229

Han, F., Wallberg, A., Webster, M.T. (2012) From where did the Western honeybee(Apis mellifera) originate? Ecol. Evol. 2, 1949-57

Hoff, P.D. (2009) A first course in Bayesian statistical methods. Springer, New York

Holmes, M.J., Allsopp, M.H., Noach-Pienaar, L.A., Wossler, T.C., Oldroyd, B.P., Beekman, M. (2010) Sperm utilization in honeybees (Apis mellifera scutellata and A. m. capensis) in South Africa. Apidologie 42, 23-28

Howard, D.J. (1993) Reinforcement: origin, dynamics, and fate of an evolutionary hypothesis. In: Harrison, R.G. (ed.) Hybrid zones and the evolutionary process, pp. 46-69. Oxford University Press, Oxford

Kerr, W.E., Bueno, D. (1970) Natural crossing between Apis mellifera adansonii and Apis mellifera ligustica. Evolution 24, 145-148

Koeniger, G., Koeniger, N., Pechhacker, H., Ruttner, F., Berg, S. (1989) Assortative mating in a mixed population of European honeybees Apis mellifera ligustica and Apis mellifera carnica. Insectes Soc. 36, 129-138

Koeniger, N., Koeniger, G. (2000) Reproductive isolation among species of the genus Apis. Apidologie 31, 313-339

Meixner, M.D., Worobik, M., Wilde, J., Fuchs, S., Koeniger, N. (2007) Apis mellifera mellifera in eastern Europe - morphometric variation and determination of its range limits. Apidologie 38, 191-197

Meixner, M.D., Costa, C., Kryger, P., Hatjina, F., Bouga, M., Ivanova, E., Büchler, R. (2010) Conserving diversity and vitality for honey bee breeding. J. Apic. Res. 49, 85-92 
Mistro, D.C., Rodrigues, L.A.D., Ferreira, W.C. (2005) The Africanized honey bee dispersal: a mathematical zoom. Bull. Math. Biol. 67, 281-312

Nazzi, F. (1992) Morphometric analysis of honey bees from an area of racial hybridisation in northeastern Italy. Apidologie 23, 89-96

Oldroyd, B.P., Cornuet, J.M., Rowe, D., Rinderer, T.E., Crozier, R.H. (1995) Racial admixture of Apis mellifera in Tasmania, Australia: similarities and differences with natural hybrid zones in Europe. Heredity 74, 315-325

Oldroyd, B.P., Reddy, M.S., Chapman, N.C., Thompson, G.J., Beekman, M. (2006) Evidence for reproductive isolation between two colour morphs of cavity nesting honey bees (Apis) in south India. Insectes Soc. 53, 428-434

Oleksa, A., Chybicki, I., Tofilski, A., Burczyk, J. (2011) Nuclear and mitochondrial patterns of introgression into native dark bees (Apis mellifera mellifera) in Poland. J. Apic. Res. 50, 116-129

Oleksa, A., Tofilski, A., Gawroński, R. (2013) Rural avenues as a refuge for feral honey bee population. J. Insect Conserv. doi:10.1007/s10841-012-9528-6

Peakall, R., Smouse, P.E. (2006) genalex 6: genetic analysis in Excel. Population genetic software for teaching and research. Mol. Ecol. Notes 6, 288-295

Phiancharoen, M., Wongsiri, S., Koeniger, N., Koeniger, G. (2004) Instrumental insemination of Apis mellifera queens with hetero- and conspecific spermatozoa results in different sperm survival. Apidologie 35, 503-511

Pritchard, J.K., Stephens, M., Donnelly, P. (2000) Inference of population structure using multilocus genotype data. Genetics 155, 945-959
Rowell, G.A., Taylor, O.R., Locke, S.J. (1986) Variation in drone mating flight times among commercial honey bee stocks. Apidologie 17, 137-158

Ruttner, F. (1988) Biogeography and taxonomy of honeybees. Springer, Berlin

Semkiw, P., Skubida, P. (2010) Evaluation of the economical aspects of Polish beekeeping. J. Apic. Sci. 54, 5-15

Schmidt, J.O. (1995) Dispersal distance and direction of reproductive european honey-bee swarms (Hymenoptera, Apidae). J. Kans. Entomol. Soc. 68, 320-325

Sheppard, W.S., Rinderer, T.E., Mazzoli, J.A., Stelzer, J.A., Shimanuki, H. (1991) Gene flow between African- and European-derived honey bee populations in Argentina. Nature 349, 782-784

Siuda, M., Wilde, J., Bratkowski, J., Chuda-Mickiewicz, B., Woyke, J., et al. (2011) Wintering queen bees in modified mating nuclei. J. Apic. Sci. 55, 87-98

Smith, R.J. (2009) Use and misuse of the reduced major axis for line-fitting. Am. J. Phys. Anthropol. 140, 476-486

Soland-Reckeweg, G., Heckel, G., Neumann, P., Fluri, P., Excoffier, L. (2009) Gene flow in admixed populations and implications for the conservation of the Western honeybee. Apis mellifera. J. Insect Conserv. 13, 317-328

Solignac, M., Vautrin, D., Baudry, E., Mougel, F., Loiseau, A., et al. (2003) Five hundred and fifty microsatellite markers for the study of the honeybee (Apis mellifera L.) genome. Genetics 167, 253-62

Tuchashvili, S.M. (1969) Flight activity of drones of different races. [In Russian]. Pchelovodstvo 10, 16-17 